\title{
Paper Metaphor for Tabletop Interaction Design
}

\author{
Guillaume Besacier ${ }^{1}$, Gaétan Rey ${ }^{1}$, Marianne Najm², Stéphanie Buisine², \\ and Frédéric Vernier ${ }^{1}$ \\ ${ }^{1}$ Limsi-cnrs, bp 133, 91403 Orsay Cedex, France \\ ${ }^{2}$ Ensam-lcpi, 151 boulevard de l'hôpital, 75013 Paris, France \\ guillaume.besacier@limsi.fr
}

\begin{abstract}
The aim of this paper is to explore new metaphors for interaction design on tabletop system. Tabletop systems are shared horizontal surface for co-located collaboration, which leads to original problems when designing interactions. We propose two metaphors based on the paper: the peeling metaphor, and the slot metaphor, and then suggest a way of using them to design new interactions for solving some of the problems of tabletop systems: documents organization, documents transmission and documents duplication.
\end{abstract}

Keyword: Tabletop, interaction design, paper metaphor.

\section{Introduction}

Nowadays revolution to mobile and miniaturized devices hides another creeping evolution toward very large surfaces usable simultaneously by more than one user, called Tabletops. Tabletop is a new form of computer, complementary to the usual desktop and laptop. It is especially efficient for performing co-located collaborative tasks. Indeed, we observe that classical computers can tend to get in the way of having the work done. In the setting of a staff meeting aiming to agree on the wording of a given document and collectively approve it, the usual solution involves a laptop per participant, each one with its own digital copy of the document. People have to handle the document versioning and updates themselves, and each modification has to be applied individually on each copy of the document. This work could be done by the computer in natural and easy-to-understand way by having a single digital copy of the document physically shared by the participants. With tabletop computers, the participants can gather around a digitally-augmented table. The table is at the same time an input device and an output device, allowing for direct manipulation of the data (using one's finger or an augmented pen). Our experiments [5] with creativity meeting show that tabletop systems increased the collaboration among users and give them a feeling of improved efficiency.

Tabletop systems introduce new challenges in user interaction design. The main challenges include:

Multiple users have to share the same surface and interact seamlessly together or in parallel. 
They are located all around the table, leading to different points of view on the documents.

Direct finger or pen interaction limits one's possibilities of interaction to one's physically reachable table space. This space can be divided according to the effort needed to access it (i.e. right in front of the user, need to get up and lean on the table, need to move across the room, etc.)

These challenges lead us to rethink the traditional desktop metaphor. To explore the new possibilities we are developing DiamondSpin [14], a toolkit to build user interfaces for tabletop systems. We invented new interactions and experienced the need for new mechanisms in graphical toolkit. Features we already implemented include color coded frames for distinction of window ownership, automatic window rotation within a polar coordinate system, relocatable and non-modal pop-up menus, push-up menubars sliding along the table edge, and free-hand annotation mechanisms.

\subsection{Paper Metaphor}

We reckon that, while the technologies like videoconferencing, digital paperboard, and the like are being introduced in the users' workplace, face-to-face pencils and paper meetings are still extremely common. Participants to such meeting intuitively perceive the interactions they can perform with a sheet of paper, a file, or a filing cabinet. In order to facilitate the transition to tabletop systems we have to offer the same intuitiveness.

At the same time we observed other new user interaction techniques based on paper metaphor $[1,2,8]$. In that context, the correspondence between sheet of paper and windows is fundamental. We think this metaphor must be extended to the way users interact with the system. Indeed, even if rotated windows look similar to real paper, the current interaction techniques to rotate them may be difficult to understand. Most of them are barely metaphoric (i.e. adding corners to the windows, and have the user touch the corners to rotate it), or can be difficult to perceive at first glance because of a lack of on screen indications or feedback (i.e. two fingers rotation).

\subsection{New Interactions}

In this paper, we present two new metaphors based on the paper: peeling and slots, and how we suggest using them to design new interaction techniques.

The peeling metaphor allows users to peel a document in a permanent way (as opposed to the temporary peeling used to see underneath the peeled document). Like with real paper, users can use this technique to reduce the space used by a window. They can also use it to access the verso of the window, where we placed a fully functional graphical user interface, and to make folders of documents in an informal way, by sliding documents between the two sides of a peeled container-document.

The slot metaphor allows the use of slots in tabletop applications. Slots are an affording place to insert paper document, like the slot of a letterbox, but are also commonly accepted as a processing place, like a CD slot where the disk is played. We use a set of slots to move documents to unreachable places on a very large tabletop system, and a special copy slot to duplicate a document. 


\section{Related Works}

Tabletop system first raised hardware challenges and different technologies were developed for tabletop hardware (vision-based [4], electric contact [7], with more than two states [9], etc.) and output system (video-projector, individual head mounted displays [3], screen sensitive to the user's position around the table [11], etc.). While finding standard application don't fit well on tabletop systems, the need for new interaction technique bring us to build the DiamondSpin[14] toolkit to allow WIMP applications to work in a tabletop world. DiamondSpin allows concurrent work on the table but barely support collaboration without specific development for document sharing. Many research works claim the WIMP approach isn't rich enough and should be extended to new interaction styles ([2], [8]) called post-WIMP or rich desktop.

Studies on tabletop interface have been numerous with the first IEEE International Workshop on Horizontal Interactive Human-Computer Systems and the special issue of Computer Graphics and Applications on Tabletop systems [10] although they tend to exemplify their interaction techniques only on static documents, with tasks like organizing pictures, or use abstract icons to figure documents [1]. In our work, indeed, we think tabletop documents can be constructed like desktop windows, by mixing standard interactors with original interactions for manipulating whole document or group of documents. Our approach is toolkit-based, with purposes similar to those of Metisse [6] for the desktop computer.

\section{Peeling}

The possibility to peel (or to fold) digital windows has been proposed as a temporary state of the window, with the purpose of seeing other windows stacked below [2], or facilitating inter-documents interactions between overlapping windows [8]. When the user releases the document or finishes the interaction which prompted for peeling, the document springs back to its unpeeled initial state.

We, on the contrary, would like to explore the possibility to peel documents definitely. It would allow the users to further interact with the peeled document, and unpeel it manually later.

Peeling interaction needs to be both obvious and natural. It needs to be obvious because it's a new interaction. If it's triggered by some obscure sub-sub-option or by a complicated movement of the finger, users will not search for it, and thus will not find it. It also needs to be natural because it's a high level interaction that can be performed on any document. It must not annoy the user while s/he is working on her/his documents.

We propose three ways to activate peeling:

- With a dedicated interactor in the document frame, for example a corner of the document, which can be dragged. This is the most obvious way: when they see an interactor they don't know yet, users may try it. On the cons side, it is not very metaphoric, and does not allow for an arbitrary peeling. The peeling corner has to be on the peeled side, while the opposite corner has to be static. 
- With a quick drag gesture from a border of the document to the inside of it. The document can be peeled in any shape the user wants. S/he could also peel a document more than once.

- Automatically, when the document hits an obstacle (the border of the table, another document, etc.). With a physic-like reaction, the document peels itself to avoid the collision with the obstacle. This technique has the advantage of not introducing a new interaction: it uses the standard interaction for moving document, thus probably reducing the learning time for the user.
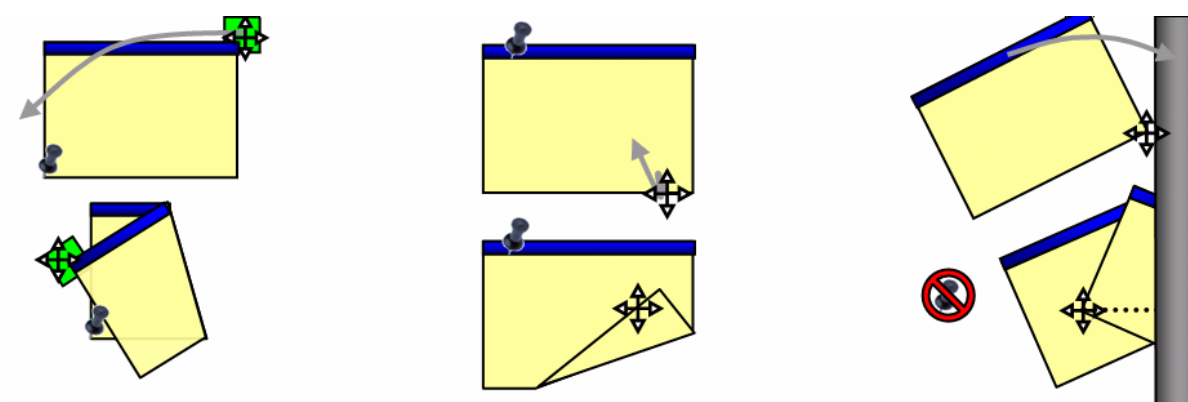

Fig. 1. Three ways for peeling documents. For each technique, the gray line indicates the user gesture, the four arrows indicate the location of the moving point on the peeled side, and the pinpoint indicate the location of the static point on the front side.

\subsection{Recto Verso Documents}

The first consequence after peeling a document is to make visible a new artifact: the back side of the document. It is empty for temporary peeling (see for example, [6] and [8]), because the user can not interact with it. S/he is already interacting with the component that caused the peeling, and if s/he stops this interaction, the document will stop being peeled. In our case, the user could interact with the peeled side. We think it would be interesting to use this space as the verso of the document. Like the verso of a sheet of paper, the verso of the document should have the same properties than the recto. As our documents are composed of GUI components, the verso of a document can also hold a fully functional graphical user interface. The user can interact with it as if it was a normal document, within the limits, of course, of the visible part of the peeled side.

We think the verso of the document is especially adapted to put the control interfaces associated with the content of the recto. Indeed, tabletop systems are not adapted to the usual pop-up dialog boxes. The link between the dialog box and the document is not visually obvious. While this is not a problem with desktop computer, where a single user works on a single foreground window, it is a problem on tabletop, where multiple users work, together or in parallel, on multiple documents. Putting the dialog boxes contents on the back of the associated document creates a strong link between them. 
We envision a translucent texture for the peeled side. The user could see a part of the document content through the peeled side, and thus see the result of his/her modifications.

\subsection{Peeling for Piling}

Grouping and classifying documents is an important task on computer systems. Many solutions have been studied, but we think they lack of freedom to improvise. In real world, we noticed piles of documents surrounded by a folded piece of paper on many desks. It leads us to imagine a way to use the peeling technique to solve the piling problem we were faced on tabletop systems.

We propose a way to fold any tabletop document in two and to use it as a handler to keep others documents together. Our main point is to allow for opportunistic creation of temporary piles. Study [12] shows that a pile is an interesting structure to support casual organization of documents.
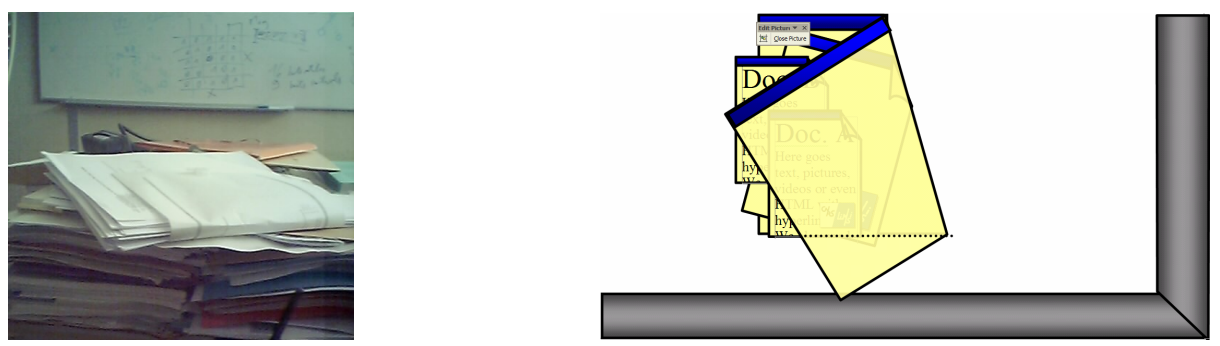

Fig. 2. On the left, a pile of papers in a folded piece of paper, used as inspiration for our metaphoric interaction technique. On the right, a design prototype with three tabletop documents held by a fourth document peeled in two.

The life cycle of a pile can be divided in two phases: the creation of the pile as a set of documents, and the manipulation of the resulting pile and of its documents. For the creation, we envision two ways of building a pile: the pile handler precedes the grouping of the documents, or the documents are grouped and then a handler is put around them. Both ways have pros and cons and will need to be experimented.

In the first case, the user first peels a document, and then slides other documents in between the two sides of it. We have to determine at what time a potential handler grabs a document. Users might want to move a document close to or over a pile without putting it in the pile. Modifying the behavior of a basic interaction (moving documents around the surface) might disturb them.

In the second case, the user first regroups his//her documents on a user-chosen spot on the table, and then peels a document that is underneath the stack. Problems of occlusion and z-order can arise. If the documents to be piled are covering the handler, the user will have difficulties to interact with the latter, especially to peel it. This technique also implies a change to the z-order management. Usually, when a user touches a document, the document moves to the top of the z-order. Here, it would need to stay at its position for the metaphor to work. Only the peeled face should be drawn top-most. 
For the second phase, manipulation of the pile, we foresee further than just keeping documents together. Indeed, for our technique to be a contribution to the user effectiveness when manipulating collection of data, we need to break the metaphor and take advantage of the digital information documents carry (document type, metadata, document content). This information could be used to quickly perform tiresome tasks on the pile. For example, all piles could be sorted by document creation or modification date, document title, type, or author. Piles containing a single type of documents could expose document-defined behavior:

- A pile of pictures could launch a slideshow or display thumbnail view of all the contained pictures.

- A pile of graded homework could compute the mean and standard deviation of the grades, display a repartition graph or sort the works by grade or student's group.

- A pile of business cards could expose advanced search functionality, allow sending a mailing to all the people is the pile, or update existing older business cards when user puts in new ones.

The pile handler being peeled in two, the verso of the pile handler is visible. Usually, we would put an interface to manipulate the document recto (that is, the pile handler). The latter having been "recycled" as a pile handler, we can reuse its verso to display the interface to manipulate the pile.

\section{Slots}

One main challenge of tabletop system is document management. As tabletop systems don't have a preferred orientation managing document doesn't only imply moving and organizing them but also orienting them. As tabletop system remain small in size and resolution much poorer than real paper a tabletop system aiming to manage large numbers of documents must allow zooming in and out of documents. With all these constraints in mind when we explored the paper metaphor we found the idea of slot very useful for document management.
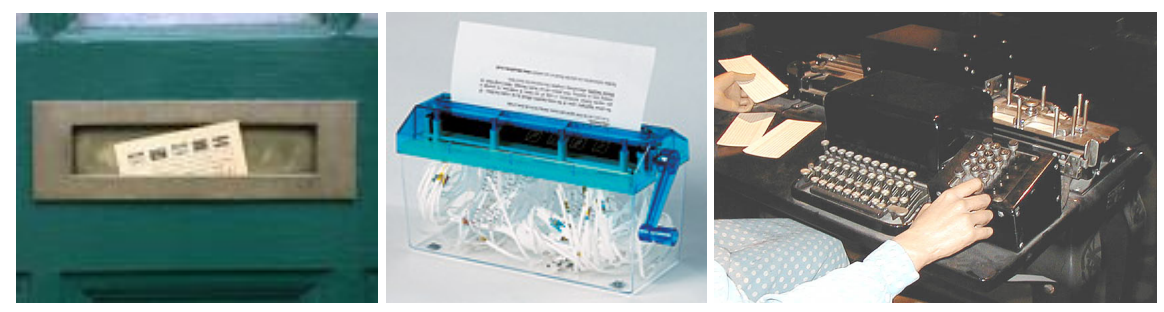

Fig. 3. A letter slot, a paper shredder and a punch card computer (IBM Type 31) used as inspirations for the slot metaphor on tabletop

\subsection{Moving Documents with Slots}

In real life, slots can be used to hide partially document while showing their presence (See Figure 3), to destroy document (Paper shredder), transmit document (fax copier), etc. 
Among all these possible facets of the slot metaphor, transmission looked very promising for tabletop systems for two reasons. First, transmitting document across two tabletop systems raise the spatial problem of addressing the document to the right person on the remote tabletop system. The second interesting problem slot can address occurs when transmitting a document locally to someone on the other side of the table. In this very common scenario we don't have a good solution when the table is very large, and even when the table is small enough orientation of the content never satisfies fully both users. As illustrated in Figure 4 we propose to use a slot to transmit a document from one side of the table to another. If the tabletop system addresses multi-touch technology, a second finger could be used in order to activate the functionality (illustrated as a finger pressing a green button), making the slot armless when a document is just moved over it. In some sense the slot acts as a Transfolder as defined by Sire [15] with a more explicit feedback.

As illustrated on the right part of Figure 4, reception can also act only on demand. What is really specific to tabletop in this technique is the ability to control how the document is received since the target is on the same display and the possibility to rotate the document on the reception side. As shown on the top right part of the figure, the document can flip at the end of transmission or even during transmission (not shown on the illustration).

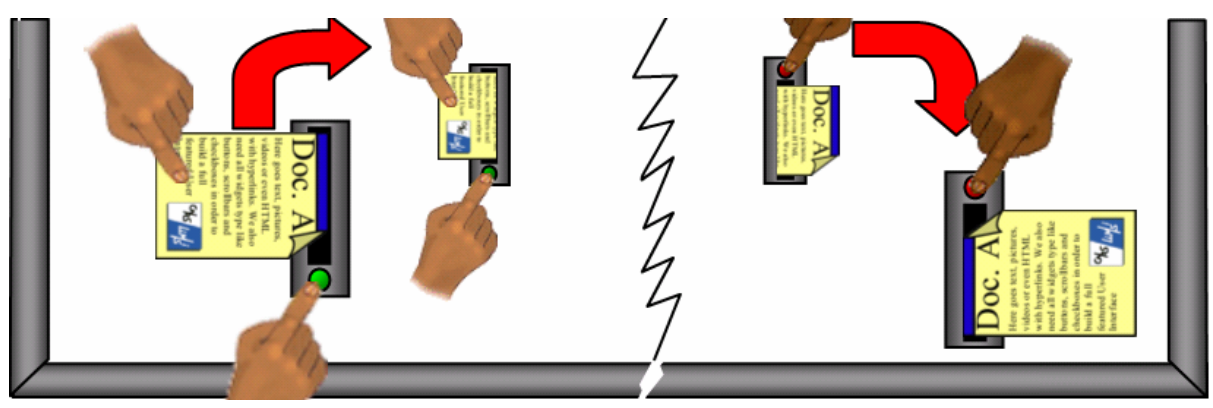

Fig. 4. Document transmission across the table with a slot

The other part of the slot metaphor we find interesting is the affordance to process document (like shredder, laminator or CD slot). An important task we could improve on tabletop system are the free-hand annotation mechanisms (as well known for TabletPC). The slot metaphor could be used to erase annotations, change their color, recall old ones, reveal colleague's annotations and so on.

In the same way an important problem with tabletop system is document sharing policies arising when multiple people start to manipulate document at the same time. A possible resolution of concurrent action is to prevent action from a second user when another user already touched the document. This approach works fine for concurrent work but need to define an explicit way to exchange document in order to also allow group work and processing a document through a slot afford an owner's change (like for letter's slot).

At last the slot metaphor may help to resolve a key challenge of tabletop systems: grouping of documents. If a slot is not fixed on the table but moveable it may serve as a convenient container like the piling technique suggested in the previous section. 


\subsection{Duplication of Documents}

Document duplication is a major element in a collaborative workspace. To facilitate duplication of document we suggest the following interaction techniques based on the paper metaphor.

Copy slot. This technique illustrated in Figure 5 is based on a copier-like slot. When inserted in the slot, a number of copies of the document come out of it. Another version of this slot may be understood as a fax metaphor when only the original document get out of the slot and copies get out of another slot.

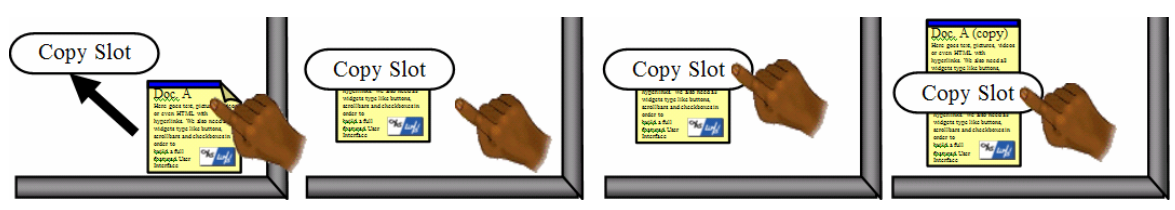

Fig. 5. Copy slot metaphor

Tearing-regeneration. Tearing regeneration differs from the previous technique because it doesn't need a dedicated slot. This metaphor uses direct manipulation to create a copy of the document. We suggest two different ways to activate the technique, by stretching and by folding, both needing two fingers.

In pulling activation, when document is pulled in two opposite directions, it first grows until a given size limit if zoomable and when a size limit is reached a crack appears. The crack acts like a generic slot with two half document out of it and notifies users the ongoing possibility to duplicate the document. If user pursues her/his action the crack totally splits the document in two and each parts finally regenerates its missing half. The same technique performed by two users could explicitly solve a conflict (two users trying to move the same document, like [13]).

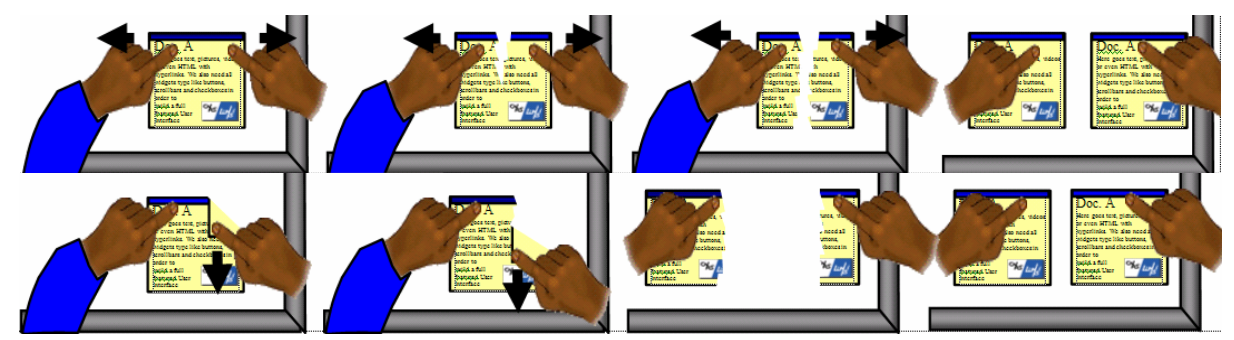

Fig. 6. Tearing-regeneration metaphor by stretching (top) and by folding (above)

Our second two-hand method is based on the folding technique presented previously. The technique occurs when a pivot point is set with a first finger while the document is folded with a second finger like illustrated in the Figure 6. When the folding line reaches the pivot point, a crack appears like in the stretching method. If the second finger takes the torn far enough to split the document in two, it regenerates missing parts again. The choice of sizes of the two parts may indicate an original and 
a copy. At Last, comparing to the previous activation mode, the feedback duration will give more opportunities to cancel the duplication.

\section{Conclusion}

In this article we investigated metaphors related to paper in order to solve tabletop centric problems like document sharing and document grouping. As tabletop systems are far more complicated than traditional desktop systems (with more than one user, more than one control point per user, multiple valid orientations around the table, unreachable part of the table, etc.), it seamed important to us to find new sources of inspiration to design metaphoric solutions compatible with the existing user experiences. Our study highlighted two specific rich metaphors we could use to design interactive techniques meeting tabletop needs: slot and peeling.

We are now going to implement these interaction techniques in our DiamondSpin toolkit[14]. These new techniques will be provided as generic tools reachable in the default menu so existing tabletop applications using DiamondSpin will take advantage of them.

Finally we will have to conduct the evaluation phase, which gives rise in itself to new research challenges. Indeed the choice of a protocol is not straightforward to catch the specificities of an interaction metaphor, to test its power, its relevance and usefulness. For example we could set up a three-step evaluation process: in the first step users would have to achieve some pretext task and would be exposed to the metaphor with no instruction on what its purpose is and how to use it. In this phase we would just observe users' behavior and collect their spontaneous comments. In the second evaluation step we would instruct the users to try and discover the target widgets: here we could study the affordance of our design elements (how do the graphical features of the widgets suggest the way to use them) by recording users' behavior and performance. We could also investigate the extent to which this affordance matches the intended metaphor by collecting users' feelings about the design and the metaphor, etc. Finally, the third evaluation step would consist in making the metaphors available to well-informed users in the context of a given task. In this phase we could study the free usage of the metaphors and examine their influence on the performance in task achievement, on the collaborative behaviors of participants and on their subjective experience.

\section{References}

1. Agarawala, A., Balakrishnan, R.: Keepin' it real: pushing the desktop metaphor with physics, piles and the pen. In: Proc. CHI 2006 pp. 1283-1292 (2006)

2. Beaudouin-Lafon, M.: Novel Interaction Techniques for Overlapping Windows. In: Proc. UIST 2001 pp. 153-154 (2001)

3. Benko, H., Ishak, E., Feiner, S.: Cross-Dimensional Gestural Interaction Techniques for Hybrid Immersive Environments. In: Proc. IEEE Virtuel Reality (VR'05) (2005)

4. Bérard, F.: The Magic Table: Computer-Vision Based Augmentation of a Whiteboard for Creative Meetings. In: Proc. PROCAMS'03 (2003) 
5. Buisine, S., Besacier, G., Najm, N., Aoussat, A., Vernier, F.: Computer-supported creativity: Evaluation of a tabletop mind-map application. HCII 2007 (2007)

6. Chapuis, O., Roussel, N.: Metisse is not a 3D desktop! In: Proc. UIST'05, pp.13-22 (2005)

7. Dietz, P., Leigh, D.: DiamondTouch: A Multi-User Touch Technology. In: Proc. UIST 2001, pp. 219-226 (2001)

8. Dragicevic, P.: Combining crossing-based and paper-based interaction paradigms for dragging and dropping between overlapping windows. In: Proc. UIST 2004, pp. 193-196 (2004)

9. Forlines, C., Shen, C., Buxton, B.: Glimpse: a novel input model for multi-level devices. Ext. Abstracts CHI 2005 pp. 1375-1378 (2005)

10. IEEE Computer Graphics and Application. Interacting with Digital Tabletops. vol. 26(5) (September/October 2006)

11. Kakehi, Y., Iida, M., Naemura, T., Shirai, Y., Matsushita, M., Ohguro, T.: Lumisight Table: Interactive View-Dependent Tabletop Display Surrounded by Mutiple Users. IEEE Computer Graphics and Applications 25(1), 48-53 (2005)

12. Mander, R., Salomon, G., Wong, YY: A Pile. Metaphor for Supporting Casual Organization of Information. In: Proc. CHI'92, pp. 627-634 (1992)

13. Ringel, M., Ryall, K., Shen, C., Forlines, C., Vernier, F.: Release, Relocate, Reorient, Resize: Fluid Techniques for Document Sharing on Multi-User Interactive Tables. In: Proc CHI 2004 pp. 1441-1444 (2004)

14. Shen, C., Vernier, F., Forlines, C., Ringel, M.: DiamondSpin: An Extensible Toolkit for Around-the-Table Interaction. In: Proc. CHI 2004, pp. 167-174 (2004)

15. Sire, S., Chatty, S., Gaspard-Boulinc, H., Colin, F.R.: How can groupware preserve our coordination skills? Designing for direct collaboration, Interact 99 (1999) 